\title{
Spontaneous Flavor and Parity Breaking with Wilson Fermions
}

\author{
Stephen Sharpe"l and Robert Singleton, Jr.f \\ Physics Department \\ Box 351560, University of Washington \\ Seattle, WA 98195, USA
}

\begin{abstract}
We discuss the phase diagram of Wilson fermions in the $m_{0}-g^{2}$ plane for twoflavor QCD. We argue that, as originally suggested by Aoki, there is a phase in which flavor and parity are spontaneously broken. Recent numerical results on the spectrum of the overlap Hamiltonian have been interpreted as evidence against Aoki's conjecture. We show that they are in fact consistent with the presence of a flavor-parity broken "Aoki phase". We also show how, as the continuum limit is approached, one can study the lattice theory using the continuum chiral Lagrangian supplemented by additional terms proportional to powers of the lattice spacing. We find that there are two possible phase structures at non-zero lattice spacing: (1) there is an Aoki phase of width $\Delta m_{0} \sim a^{3}$ with two massless Goldstone pions; (2) there is no symmetry breaking, and all three pions have an equal non-vanishing mass of order $a$. Present numerical evidence suggests that the former option is realized for Wilson fermions. Our analysis then predicts the form of the pion masses and the flavor-parity breaking condensate within the Aoki phase. Our analysis also applies for non-perturbatively improved Wilson fermions.
\end{abstract}

$\mathrm{UW} / \mathrm{PT}$ 98-2

hep-lat/9804028

Typeset in $\mathrm{LaTEX}_{\mathrm{E}}$

†sharpe@phys.washington.edu

‡bobs@terrapin.phys.washington.edu 


\section{INTRODUCTION}

This paper concerns the phase diagram of Wilson fermions (and improved versions thereof) at non-zero lattice spacing, and the restoration of chiral symmetry in the continuum limit. Some time ago, Aoki proposed a phase diagram in which there were regions of spontaneous flavor and parity breaking (which we refer to as Aoki phases) [1]. This suggestion, sketched in Fig. 1, provides a dynamical explanation for the masslessness of the pion on the lattice. Analytical and numerical support for this proposal has been given in Refs. [1 5].

The validity of this picture has, however, been challenged recently by Bitar, Heller and Narayanan [6]. These authors argue that flavor and parity violation do not occur at nonzero lattice spacing, and that, by analogy with the massless continuum theory, one should interpret the Aoki phases as containing massless quarks and exhibiting spontaneous chiral symmetry breaking. Numerical results supporting this claim are given in Refs. [7,8].

In this paper we present two arguments supporting Aoki's suggestion of spontaneous flavor and parity breaking at non-zero lattice spacing. Our first observation is that the numerical results of Refs. [7,8] are not only consistent with Aoki's picture, but they are inconsistent with the interpretation of Bitar et al.. Thus all numerical results to date are consistent with Aoki's proposed symmetry breaking.

Our second and more important observation is that, close to the continuum limit, one can study the pattern of symmetry breaking theoretically, using the chiral Lagrangian. One must augment the usual continuum chiral Lagrangian with terms corresponding to the explicit breaking of chiral symmetry at non-zero lattice spacing. The form of these terms is dictated by the symmetries of the lattice theory. Their coefficients are, however, undetermined aside from their order of magnitude. Nevertheless, it turns out that this is sufficient information to determine the pattern of symmetry breaking at small but non-zero lattice spacing, up to a two-fold ambiguity. If the sign of a particular coefficient is positive, then there is an Aoki phase with all the expected properties. If the sign is negative, then there is no Aokiphase, flavor is always unbroken, and the pions do not become massless for any value of the bare mass. The present numerical evidence suggests that the first option correctly describes (unimproved) Wilson fermions. If this is the case, our calculation makes several predictions that can be tested by numerical simulations.

In Aoki's picture, the flavor-parity violating phases shrink rapidly to isolated points as one approaches the continuum limit (see Fig. 1). At these points, the quarks are massless, chiral-symmetry is spontaneously broken, and all pions are massless. Our analysis finds that the width of the Aoki phase shrinks as $\Delta m_{0} \propto a^{3}$, up to logarithmic corrections. Perhaps more importantly, we can see how the flavor-parity breaking at non-zero lattice spacing transforms smoothly into chiral symmetry breaking in the continuum limit. In our view, the interpretation of Bitar et al. applies only in this limit.

Similar observations concerning symmetry breaking have been made previously by Creutz [9]. He performs a qualitative analysis using the linear sigma-model, and finds the same two possible patterns of symmetry breaking at finite lattice spacing. Our calculation extends his by providing a firmer theoretical basis for his observations and by making quantitative predictions.

The outline of this paper is as follows. In the next section we review Aoki's proposal for the phase diagram, and then describe the alternative view of Bitar et al.. In Sec. III we 
explain how the numerical results obtained to date are all consistent with Aoki's proposal. We then present, in Sec. IV], our Chiral Lagrangian analysis. Sec. V contains our conclusions. We collect technical issues in two appendices.

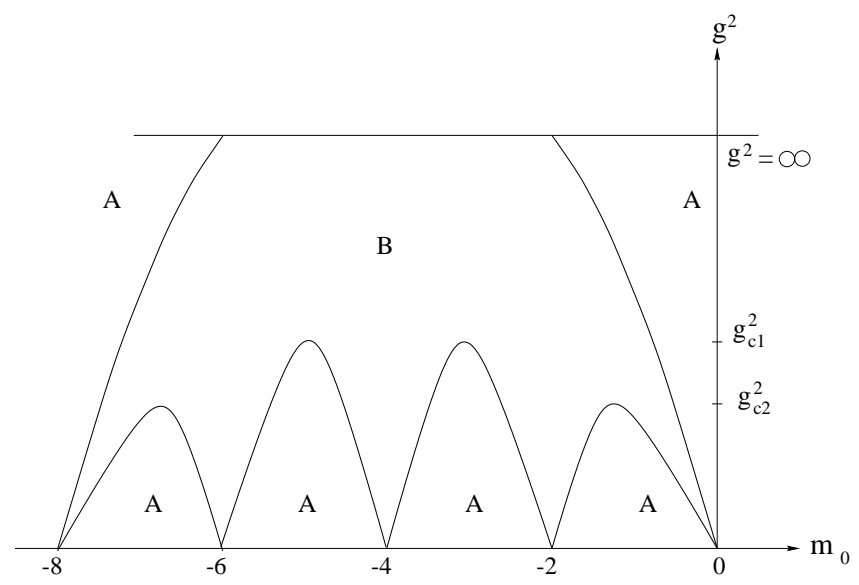

FIG. 1. The phase diagram proposed by Aoki: $g$ is the gauge coupling and $m_{0}$ the dimensionless bare quark-mass. The continuum-like phases are labeled A, and the flavor and parity broken phase B. The phase diagram is symmetric under $m_{0} \leftrightarrow-\left(m_{0}+8\right)$. The continuum limit of particular interest is that at $m_{0}=0, g=0$.

\section{REVIEW OF PREVIOUS RESULTS}

We consider QCD with two degenerate quarks, for which the fermionic part of the Euclidean lattice action is

$$
\mathcal{L}_{f}=\bar{\psi} W\left(m_{0}\right) \psi
$$

where the flavor indices are implicit, and $W\left(m_{0}\right)$ is the Wilson-Dirac operator with a (dimensionless) bare mass $m_{0}$ common to both flavors. The bare mass is related to the usual hopping parameter by $m_{0}=1 /(2 \kappa)-4$. The action is invariant under $S U(2)$ flavor transformations, under parity, and under the interchange $m_{0} \rightarrow-\left(m_{0}+8\right)$, but it explicitly breaks all axial symmetries for any value of $m_{0}$.

We are concerned here with the phase diagram in the $m_{0}-g^{2}$ plane, where $g$ is the gauge coupling. Based on perturbation theory, we expect to be able to take a continuum limit by simultaneously sending $g^{2} \rightarrow 0$ and $m_{0} \rightarrow 0$. One can, however, obtain different continuum limits by sending $m_{0}$ to any one of the values $0,-2,-4,-6$, or -8 , for which there are respectively 1, 4, 6, 4 and 1 continuum fermions. In the following we will mainly be interested in the standard continuum limit at $m_{0}=0$ (or the equivalent point at $m_{0}=-8$ ). If this point is approached in such a way that the physical quark-mass is small compared to $\Lambda_{\mathrm{QCD}}$, then we expect the theory to spontaneously break chiral symmetry and for there to be a degenerate triplet of light pions. This should be true whether we approach from above or below $m_{0}=0$, since in the continuum the sign of the quark mass is irrelevant. Similar phenomena should occur at the other continuum points, except that the symmetry group in the continuum limit will be larger $\left(S U(8)\right.$ at $m_{0}=-2$ and -6 , and $S U(12)$ at $\left.m_{0}=-4\right)$, 
and so there should be correspondingly more pions. I In fact, it may be that the dynamics with these large numbers of flavors is different from the $m_{0}=0$ and -8 cases, since above a critical number of flavors we expect there to be no chiral symmetry breaking and no confinement [10].

We are particularly interested in how the phenomena associated with chiral symmetry breaking emerge in the continuum limit, given that the lattice theory has no such symmetry. In particular, if the continuum limit is approached so that the quarks are massless, then the resulting theory should contain massless Goldstone pions. The standard view of how the theory accomplishes this is that there is a critical line, $m_{c}\left(g^{2}\right)$, along which the triplet of pions is massless. This line runs from $m_{0}=-2$ at $g^{2}=\infty$ to the continuum point at $m_{0}=0$, $g^{2}=0$. At non-zero $g^{2}$, the pions are not Goldstone particles associated with spontaneous chiral symmetry breaking (and in particular their interactions are not restricted by chiral symmetry), but they become so in the continuum limit.

\section{A. Aoki's proposal}

Based on a variety of considerations, Aoki proposed that the vanishing of the pion mass is associated with the spontaneous breakdown of flavor symmetry [1]. His proposal for the phase diagram is sketched in Fig. 1. The phases labeled A are "continuum-like" in the sense that the $S U(2)$ flavor symmetry is unbroken. In phase B, by contrast, flavor symmetry is spontaneously broken down to $U(1)$, and parity is also spontaneously broken. There are thus two Goldstone bosons in this phase, which are exactly massless in infinite volume, even though the lattice spacing is non-zero. We will call these the charged pions. The neutral pion which completes the flavor triplet is not a Goldstone particle, and is thus massive within phase B. The transitions between the two phases are continuous, so that as one approaches the boundary from within phase $\mathrm{B}$, the neutral pion must also become massless so as to restore flavor symmetry. This means that as one approaches the boundary from within phase A, the degenerate triplet of pions becomes massless. The critical line discussed above is the rightmost phase boundary.

This proposal has been established at strong coupling [1], for which there are just two critical lines (related by the symmetry $\left.m_{0} \rightarrow-\left(m_{0}+8\right)\right)$. At weak coupling, if one is to reproduce the continuum limits expected from perturbation theory, then one must introduce the additional regions of phase A shown in the figurel. In particular, if the continuum limit at $m_{0}=0$ can be approached from positive and negative mass, then the width of phase B must vanish at $g^{2}=0$. Aoki argues that, since phase B is not observed in perturbation theory,

\footnotetext{
*At non-zero lattice spacing, however, only an $S U(2)$ subgroup of the full continuum flavor symmetry is exact, so the full pion multiplet will be broken into representations of this subgroup, with the lightest pions being in a triplet.

${ }^{\dagger}$ These new regions of phase A appear below critical values of $g^{2}$, denoted $g_{c 1}^{2}$ and $g_{c 2}^{2}$ in the figure. These are conventionally drawn as being equal, but we can see no reason to expect this equality. The particular ordering of $g_{c 1}^{2}$ and $g_{c 2}^{2}$ shown in the figure is an arbitrary choice.
} 
its width must be a non-perturbative function of $g^{2}$, i.e. have a power law dependence on $a$. We argue in Sec. IV that the dependence is $\Delta m_{0} \propto a^{3}$ (up to logarithms).

To investigate the spontaneous symmetry breaking in phase B, Aoki added to the Lagrangian (2.1) a source term that explicitly breaks both flavor and parity,

$$
\mathcal{L}_{\text {source }}=i \hbar \bar{\psi} \gamma_{5} \sigma_{3} \psi
$$

with $\sigma_{3}$ acting in flavor space. Flavor and parity are spontaneously broken if, in the limit that the source is removed, a condensate remains:

$$
\lim _{h \rightarrow 0^{ \pm}}\left\langle i \bar{\psi} \gamma_{5} \sigma_{3} \psi\right\rangle= \pm \text { const }
$$

Flavor rotations in the 1- and 2-directions change the condensate, while those in the 3direction do not. The symmetry is thus broken from $S U(2) \rightarrow U(1)$, with the condensates inhabiting the coset space $S U(2) / U(1)$. The condensate (2.3) appears to violate theorems by Vafa and Witten which state that vector-like theories do not spontaneously break flavor [11] or parity [12]. As explained in Appendix A, however, these theorems are not applicable to the case at hand.

Numerical evidence supporting Aoki's proposal has been given in Refs. [1 5]. In particular, Ref. [1] studied the pion mass as a function of $m_{0}$ at $g^{2}=1\left(\beta=6 / g^{2}=6\right)$ in the quenched approximation, finding evidence for all 10 critical lines predicted near the continuum limit (although this required the removal by hand of so called "exceptional configurations"). Unquenched simulations in small volumes also found evidence of Aoki phases, although Ref. [5] did not find them at the smallest couplings studied. This could, however, be due to the small width of the phases.

\section{B. The proposal of Bitar, Heller and Narayanan}

The phase diagram for Wilson fermions has recently been reconsidered in Refs. [6 8]. In Ref. [6], Bitar et al. take issue with Aoki's proposal. They point out that, in the massless limit of the continuum theory, a condensate of the form of (2.3) is simply an axial rotation of the usual flavor diagonal condensate, and thus breaks neither flavor nor parity. In other words, the condensate (2.3) spontaneously breaks the chiral group $S U(2)_{L} \times S U(2)_{R}$ down to an $S U(2)$ subgroup, albeit a different subgroup from the usual flavor group (its generators are vector rotations in the 3 -direction and axial rotations in the 1 - and 2 - directions). This unbroken subgroup can be defined to be the flavor symmetry. Similarly, the condensate preserves a discrete symmetry, which is the original parity transformation conjugated by an axial rotation, and this combined symmetry can be taken to be parity. Based on these observations, they argue that the Aoki phase should be interpreted as having massless quarks and spontaneous chiral symmetry breaking. The condensate of (2.3) should be interpreted as (an axial rotation of) the usual chiral condensate. Flavor and parity symmetry, they claim, are not broken at non-zero lattice spacing.

To study the issue numerically, Refs. [7,8] investigate the spectrum of the overlap Hamil- 
tonian $H\left(-m_{0}\right)=\gamma_{5} W\left(m_{0}\right) .7$ The advantage of this operator over the Wilson-Dirac operator is that it is Hermitian, and so its eigenvalues are confined to the real axis. This allows one to define a spectral density $\rho\left(\lambda ; m_{0}\right)$ both on the lattice and in the continuum. As explained in Refs. [7],8], the central issue is whether this spectral density, considered as a function of $\lambda$ for fixed $m_{0}$, vanishes for a region around $\lambda=0$. In other words, does the spectrum have a gap? This is important because, as we now explain, the absence of a gap is indicative of chiral symmetry breaking in the continuum.

We begin by recalling that the Dirac operator in the continuum, unlike the Wilson-Dirac operator on the lattice, has a spectrum confined to the imaginary axis. Thus one can define a spectral density $\tilde{\rho}(\lambda)$ for the operator $-i \not D$. As first noted by Banks and Casher [13], chiral symmetry breaking occurs if $\tilde{\rho}(\lambda)$ does not vanish at $\lambda=0$. In the continuum, the spectrum of the overlap Hamiltonian, which is just $\gamma_{5}(\not D+m)$, is related to that of $-i \not D$ by

$$
\rho(\lambda ; m)=\left\{\begin{array}{cl}
\frac{|\lambda|}{\sqrt{\lambda^{2}-m^{2}}} \tilde{\rho}\left(\sqrt{\lambda^{2}-m^{2}}\right) & |\lambda|>|m| \\
0 & |\lambda| \leq|m|
\end{array} .\right.
$$

We see that at non-zero quark-mass, $\rho(\lambda ; m)$ has a gap between $\lambda=-|m|$ and $|m|$. As the quark mass $m \rightarrow 0$, note that $\rho(\lambda ; m)$ converges to $\tilde{\rho}(\lambda)$, albeit non-uniformly. Thus the gap closes in the chiral limit and $\rho(\lambda ; 0)=\tilde{\rho}(|\lambda|)$. Based on this continuum result, Ref. [6] argues that, if the spectrum of $\gamma_{5} W\left(m_{0}\right)$ has no gap, then one should interpret the lattice theory as having massless quarks and chiral symmetry breaking.

As noted in Ref. [6], the spectral density at zero eigenvalue is related to the condensate (2.3) proposed by Aoki,

$$
\lim _{h \rightarrow 0^{ \pm}}\left\langle i \bar{\psi} \gamma_{5} \sigma_{3} \psi\right\rangle=\mp 2 \pi \rho\left(0 ; m_{0}\right)
$$

Thus Bitar et al. do expect that the Aoki phases should be characterized by a condensate, as well as vanishing pion masses.

References [0,8] study the low-lying spectrum of $\gamma_{5} W\left(m_{0}\right)$ for the mass range $-2 \leq$ $m_{0} \leq 0$. They have results on a variety of gauge field ensembles (quenched with and without improvement, and partially quenched) all with $g^{2} \approx 1$. They find that in all cases the gap in the spectrum closes for bare masses below a critical line, i.e. for $m_{0}<m_{1}\left(g^{2}\right)$. This lies very close to the critical curve $m_{c}\left(g^{2}\right)$ determined from the vanishing of the extrapolated pion mass. We follow Ref. [8] and assume that the curves would coincide were one to extrapolate including the effects of quenched chiral logarithms. What is perhaps unexpected, however,

${ }^{\ddagger}$ The sign choice of $m_{0}$ is the convention introduced in Refs. [17] and adopted also in Refs. [6] [8].

${ }^{\S}$ Spectral densities can be defined on a given gauge configuration by taking the infinite volume limit. We refer to these as $\rho_{A}\left(\lambda ; m_{0}\right)$ in the continuum, and $\rho_{U}\left(\lambda ; m_{0}\right)$ on the lattice, with the proviso that on the lattice we average over a configuration and its parity conjugate. The quantity of interest here, $\rho\left(\lambda ; m_{0}\right)$, is obtained by averaging over all configurations, with the appropriate weights. For further discussion see Appendix B. 
is that the gap does not open up before they reach $m_{0}=-2$. This is inconsistent with the results of Ref. [4], who find that the Aoki phase has a width of $\Delta m_{0} \approx 0.1$.

\section{CONSISTENCY}

In this section we address the conflict between the interpretations of Bitar et al. and Aoki. We begin with points of agreement. Both contend that there are two phases, and that phase $\mathrm{B}$ is characterized by a condensate of the form (2.3). They also agree that the masses of all three pions vanish along the boundaries between phases. The conflict concerns the number of massless pions within phase $\mathrm{B}$ and the interpretation of the condensate.

We think that Aoki's proposal is a correct description of what happens at any non-zero lattice spacing, while the interpretation of Bitar et al. is an approximate description that becomes valid in the continuum limit. In this section we elaborate on the former point, while in the next we present a framework within which to understand the latter.

The essential point has already been made by Aoki [1]. The only exact symmetry of the lattice theory is the $S U(2)$ flavor symmetry, and the condensate (2.3) spontaneously breaks this symmetry down to $U(1)$. Thus Goldstone's theorem implies that there are only two massless pions in the broken phase.

It is worthwhile discussing the symmetry breaking in more detail, so as to emphasize the differences between the massless continuum theory and the lattice in phase B. To study spontaneous symmetry breaking, one adds a source term that explicitly breaks the symmetry, calculates the order parameter (here the condensate), and then takes the limit of vanishing source strength. If the symmetry is spontaneously broken from $G \rightarrow H$, the resulting condensate lives in the coset space $G / H$. One can thus explore the coset space by examining the condensate for sources that effect breaking to different $H$-subgroups of $G$. This leads us to consider, both on the lattice and in the continuum, the following source:

$$
\mathcal{L}_{\text {source }}(\boldsymbol{\theta}, h)=h \bar{\psi} \exp \left[i \boldsymbol{\theta} \cdot \boldsymbol{\sigma} \gamma_{5}\right] \psi,
$$

where $\boldsymbol{\sigma}$ is a three-vector of Pauli matrices that acts only in flavor space. This source is obtained by applying a general chiral transformation to the mass term $h \bar{\psi} \psi$. We will denote the norm of $\boldsymbol{\theta}$ by $\theta$, while $\hat{\boldsymbol{\theta}}$ will represent a unit vector in the direction of $\boldsymbol{\theta}$. Without loss of generality we can take $h>0$ and $0 \leq \theta<\pi$ (so that $\sin \theta \geq 0$ ). In the massless continuum theory, standard arguments presented in Appendix B lead to

$$
\begin{aligned}
\lim _{h \rightarrow 0^{+}}\left\langle\bar{\psi}_{b} \psi_{a}\right\rangle & =-\pi \tilde{\rho}(0) \cos \theta \delta_{a b} \\
\lim _{h \rightarrow 0^{+}}\left\langle\bar{\psi}_{b} i \gamma_{5} \psi_{a}\right\rangle & =-\pi \tilde{\rho}(0) \sin \theta[\hat{\boldsymbol{\theta}} \cdot \boldsymbol{\sigma}]_{a b} .
\end{aligned}
$$

where $a$ and $b$ are flavor indices, and $\tilde{\rho}(\lambda)=\rho(\lambda ; m=0)$ is the spectral density of the Dirac operator introduced above. If chiral symmetry is broken down to an $S U(2)$ subgroup, then the condensates live in $S U(2)_{L} \times S U(2)_{R} / S U(2)$, and should be described by three parameters. This is indeed what we find.

The calculation in the lattice theory is somewhat different, and is explained in Ap-

pendix B. Only the pseudoscalar condensate is proportional to the spectral density, and we find 


$$
\lim _{h \rightarrow 0^{+}}\left\langle\bar{\psi}_{b} i \gamma_{5} \psi_{a}\right\rangle=\left\{\begin{array}{cc}
-\pi \rho\left(0 ; m_{0}\right)[\hat{\boldsymbol{\theta}} \cdot \boldsymbol{\sigma}]_{a b} & \theta>0 \\
0 & \theta=0
\end{array} .\right.
$$

Equation (2.5) is a particular case of this general result. The essential difference between (3.3) and the corresponding continuum result (3.2b) is the absence of the factor $\sin \theta$. This means that the lattice condensate is of fixed magnitude, and varies in direction according to the applied source. It depends on only two parameters, the angles in $\hat{\boldsymbol{\theta}}$, and maps out the coset space $S U(2) / U(1)$. This clearly represents a different pattern of symmetry breaking than in the massless continuum theory.

We wish to emphasize that these two different modes of spontaneous symmetry breaking may be differentiated by simply enumerating the number of massless pions: in the massless continuum theory, there are three massless pions, while on the lattice in phase B there are only two. To test this, one needs to know which interpolating fields couple to the different pions. For definiteness we consider a source with $\theta=\pi / 2$ in the 3 -direction, i.e. $\boldsymbol{\theta}=\frac{\pi}{2} \hat{\boldsymbol{z}}$, which leads to the condensate $\left\langle\bar{\psi} i \gamma_{5} \sigma_{3} \psi\right\rangle \neq 0$. This is the choice used in the numerical work described in the previous section. Interpolating fields for the Goldstone pions are obtained by doing a spatially dependent infinitesimal $G / H$ transformation on the condensate. In the massless continuum theory, these transformations are the vector transformations in the 1- and 2-directions, $\psi(x) \longrightarrow \exp \left(-i \alpha(x) \sigma_{1,2}\right) \psi(x)$ and the axial transformation in the 3direction, $\psi(x) \longrightarrow \exp \left(-i \alpha(x) \sigma_{3} \gamma_{5}\right) \psi(x)$. These yield the pion fields

$$
\pi_{1}(x)=i \bar{\psi}(x) \sigma_{2} \gamma_{5} \psi(x), \quad \pi_{2}(x)=-i \bar{\psi}(x) \sigma_{1} \gamma_{5} \psi(x), \quad \pi_{3}(x)=\bar{\psi}(x) \psi(x)
$$

On the lattice axial transformations are not a symmetry, and only the first two interpolating fields correspond to Goldstone modes. We see that the charged pions are created by the familiar pseudoscalar flavor non-singlet operators, although with the flavor indices 1 and 2 interchanged. The two-point functions of such operators have no disconnected contributions, even in the presence of the source term. These are the pions whose masses have been calculated in numerical simulations.

The neutral pion, on the other hand, is created by an operator that appears to be a scalar and a flavor singlet. This is because the condensate itself points in a flavor and parity violating direction. Indeed, when calculating the neutral pion correlator it is crucial to do so with the source turned on, so as to pick out the vacuum state, and then to take the limit of vanishing source strength. The correlator for this operator has not been studied numerically. We expect such a calculation will be difficult, because it contains both disconnected and connected contractions,

$$
\left\langle\pi_{3}(x) \pi_{3}(y)\right\rangle_{U}=\operatorname{Tr} S_{U}(x ; x) \operatorname{Tr} S_{U}(y ; y)-\operatorname{Tr}\left\{S_{U}(x ; y) S_{U}(y ; x)\right\}
$$

Here $S_{U}(x ; y)$ is the fermion propagator in the presence of the source. While in the continuum, the disconnected contributions can be shown to vanish because of chiral symmetry this must be so since the three pions are degenerate - this is not so at non-zero lattice spacing. One might hope, however, that their contribution will be small close to the continuum limit, and so it would be interesting to calculate the connected contribution alone. In the next section we give a prediction for the mass of this particle in the Aoki phase. 


\section{CHIRAL LAGRANGIAN ANALYSIS}

In this section we study the extent to which the spontaneous breaking of flavor and parity at non-zero lattice spacing can be viewed as an approximation to the spontaneous breaking of chiral symmetry in the continuum limit. We do so by first determining the effective continuum Lagrangian which describes the lattice theory at non-zero lattice spacing, and then extracting the effective chiral Lagrangian which describes the long distance behavior of this effective continuum theory. The analysis relies solely on symmetries and the assumption that dimensionful quantities have a size determined by $\Lambda_{\mathrm{QCD}}$ to the appropriate power. In the following we shall, for the sake of brevity, drop the subscript on $\Lambda_{\mathrm{QCD}}$.

Close to the continuum limit, the lattice theory can be described by an effective continuum Lagrangian in which the usual terms have been supplemented by contributions proportional to powers of the lattice spacing [14]. These additional pieces are constrained by the symmetries of the lattice action, which in the present case means that they need not respect chiral symmetry. The enumeration of operators is identical to that carried out as part of the improvement program [15], and the result is

$$
\begin{aligned}
\mathcal{L}_{\mathrm{eff}} \sim & \mathcal{L}_{\mathrm{g}}+\bar{\psi}\left(\not D+\frac{m_{0}}{a}\right) \psi-\frac{\tilde{m}_{c}}{a} \bar{\psi} \psi+b_{1} a \bar{\psi} i \sigma_{\mu \nu} F_{\mu \nu} \psi+ \\
& +b_{2} a \bar{\psi}(\not D+m)^{2} \psi+b_{3} a m \bar{\psi}(\not D+m) \psi+b_{4} a m \mathcal{L}_{\mathrm{g}}+b_{5} a m^{2} \bar{\psi} \psi+O\left(a^{2}\right)
\end{aligned}
$$

where $\mathcal{L}_{\mathrm{g}}$ is the gluon Lagrangian, and $m$ is a physical mass defined below. The symbol $\sim$ indicates that we are not attempting to control factors of order unity. For example, the dimensionless lattice bare mass $m_{0}$ appears in $\mathcal{L}_{\text {eff }}$ multiplied by a factor $Z_{m}\left(g^{2}, \ln a\right) \approx 1$, which we ignore. The dimensionless constants $\tilde{m}_{c}$ and $b_{i}$ are functions of $g(a)^{2}$, and in general, also of $\ln a$. We work at a fixed $a$ and thus suppress this dependence.

The first two terms in (4.1) are the naive continuum limit of the lattice Lagrangian, while subsequent contributions result from the fact that the lattice action breaks chiral symmetry. The dominant correction at small $a$ is the additive mass renormalization proportional to $\tilde{m}_{c}$, which is linearly divergent as $a \rightarrow 0$. We can combine the two mass terms in the usual way by introducing the physical mass $m=\left(m_{0}-\tilde{m}_{c}\right) / a$ (again ignoring $Z$-factors with logarithmic dependence on $a$ ). As we will see, $\tilde{m}_{c}$ is very close to, but slightly different from, the critical mass $m_{c}\left(g^{2}\right)$ at which the pion masses vanish.

The Lagrangian can be further simplified as follows. By changing quark variables, one can remove terms that vanish by the leading order equations of motion [16], which in this case are those proportional to $b_{2}$ and $b_{3}$. Note that the $b_{4}$-term renders the effective continuum coupling constant dependent upon the bare quark-mass, and the $b_{5}$-term causes the physical mass to have a quadratic dependence on the bare quark-mass. While these two contributions cannot be removed, they can be ignored. As will become clear shortly, we focus on the region in which $a m \sim(a \Lambda)^{2}$, i.e. physical quark-masses of $O(a)$. In this region, corrections proportional to $a m$ are suppressed by $a \Lambda \ll 1$ relative to the terms we keep. With these simplifications, the effective Lagrangian becomes

$$
\mathcal{L}_{\text {eff }} \sim \mathcal{L}_{\mathrm{g}}+\bar{\psi}(\not D+m) \psi+b_{1} a \bar{\psi} i \sigma_{\mu \nu} F_{\mu \nu} \psi+O\left(a^{2}\right)
$$

i.e. QCD with a Pauli term. 
The next step is to write a generalization of the continuum chiral Lagrangian that includes the effects of the Pauli term. Without either the mass term or the Pauli term, the theory is invariant under $S U(2)_{L} \times S U(2)_{R}$ chiral rotations, and its low momentum dynamics is described by the chiral Lagrangian

$$
\mathcal{L}_{\chi}=\frac{f_{\pi}^{2}}{4} \operatorname{Tr}\left(\partial^{\mu} \Sigma^{\dagger} \partial_{\mu} \Sigma\right)
$$

Here $\Sigma$ is an $S U(2)$ matrix-valued field that transforms under the chiral group as

$$
\Sigma \rightarrow L \Sigma R^{\dagger}
$$

with $L$ are $R$ being independent $S U(2)$ rotations. Its vacuum expectation value, $\Sigma_{0}=\langle\Sigma\rangle$, breaks the chiral symmetry down to an $S U(2)$ subgroup. The fluctuations around $\Sigma_{0}$ correspond to the Goldstone-bosons,

$$
\Sigma=\Sigma_{0} \exp \left\{i \sum_{a=1}^{3} \pi_{a} \sigma_{a} / f_{\pi}\right\} .
$$

Adding the mass term to the underlying Lagrangian explicitly breaks chiral symmetry, and its contributions to $\mathcal{L}_{\chi}$ can be determined using a standard spurion analysis. Since we are only interested in determining the vacuum state, we do not consider derivative interactions. Working to second order in $m$, the potential energy is

$$
\mathcal{V}_{\chi}=-\frac{c_{1}}{4} \operatorname{Tr}\left(\Sigma+\Sigma^{\dagger}\right)+\frac{c_{2}}{16}\left\{\operatorname{Tr}\left(\Sigma+\Sigma^{\dagger}\right)\right\}^{2}
$$

with $c_{1} \sim m \Lambda^{3}$ and $c_{2} \sim m^{2} \Lambda^{2}$. We have used the property that any term invariant under the vector symmetry can be written as a function of $\operatorname{Tr}\left(\Sigma+\Sigma^{\dagger}\right)$.

We now include the Pauli term. This is straightforward since it transforms under chiral rotations exactly as does the mass term. Thus its effects can be included (along with those of the mass term) by making the substitution $m \rightarrow m+a \Lambda^{2}$. The factors of $\Lambda$ are required by dimensional analysis, and we recall that we are dropping dimensionless coefficients of order unity. Thus the potential is given by (4.6), with coefficients

$$
c_{1} \sim m \Lambda^{3}+a \Lambda^{5}, \quad c_{2} \sim m^{2} \Lambda^{2}+m a \Lambda^{4}+a^{2} \Lambda^{6} .
$$

Note that choosing $a m \sim(a \Lambda)^{2}$ makes the contributions of the quark mass term and the Pauli term comparable. This is as we would expect: if the physical mass is of $O(a)$, then the strength of chiral symmetry breaking due to the mass term should be comparable to that due to discretization.

Since we are keeping terms of $O\left(a^{2}\right)$ in $c_{2}$, we must also consider corrections of $O\left(a^{2}\right)$ to the underlying QCD Lagrangian. Fermion bilinears of $O\left(a^{2}\right)$ such as $a^{2} \bar{\psi} D^{3} \psi$ are necessarily flavor singlets, and so contribute in the same way as the Pauli term, but suppressed by $a \Lambda$. Four fermion operators such as $a^{2} \bar{\psi} \psi \bar{\psi} \psi$ can contribute directly to the $c_{2}$ term, and give an additional contribution proportional to $a^{2} \Lambda^{6}$. Thus the estimates of (4.7) remain valid. There are of course subleading contributions to these coefficients, suppressed by powers of $a \Lambda$. 
In the following we determine the properties of the chiral theory, and in particular the pattern of symmetry breaking, as a function of the coefficients $c_{1}$ and $c_{2}$. Since these coefficients depend on $m_{0}=\tilde{m}_{c}+m a$ and on $a$, we can map our results back onto the phase diagram in the $m_{0}-g(a)^{2}$ plane. We distinguish three regions of quark masses, each successively smaller by a factor of $a \Lambda$ :

1. Physical masses, i.e. $m / \Lambda$ fixed and small. For such masses, as $a \rightarrow 0, c_{1} \sim m \Lambda^{3}$ and $c_{2} / c_{1} \sim m / \Lambda$. In this case both the discretization errors and the contribution of the $c_{2}$ term can be ignored, and the symmetry breaking is as in the continuum.

2. Generic masses of $O(a)$, i.e. $a m \sim(a \Lambda)^{2}$. For these, the contribution of the $c_{2}$ term is suppressed by $a \Lambda$, but discretization errors are important in $c_{1}$. The value of $m$ at which the extrapolated pion mass vanishes is shifted by $\sim a \Lambda^{2}$ (corresponding to a shift of $O\left(a^{2} \Lambda^{2}\right)$ in $\left.m_{0}\right)$. It is useful to introduce a shifted mass, $m^{\prime}=m-a \Lambda^{2}$, (where, we stress, all constants have been set to unity), defined so that $c_{1}$ vanishes when $m^{\prime}=0$. In terms of this new mass the coefficients are

$$
c_{1} \sim m^{\prime} \Lambda^{3}, \quad c_{2} \sim m^{\prime 2} \Lambda^{2}+m^{\prime} a \Lambda^{4}+a^{2} \Lambda^{6} .
$$

3. Shifted masses of $O\left(a^{2}\right)$, i.e. $a m^{\prime} \sim(a \Lambda)^{3}$. For such masses, the coefficients can be simplified to

$$
c_{1} \sim m^{\prime} \Lambda^{3}, \quad c_{2} \sim a^{2} \Lambda^{6}
$$

The crucial point is that $c_{1} \sim c_{2}$, and so the two terms in the potential are comparable. As explained below, competition between the two terms can lead to Aoki phases.

It is immediately apparent from this discussion that the width of the region in which new phenomena can occur, due to competition between the two terms in the potential, is $\Delta m_{0} \sim a \Delta m^{\prime} \sim(a \Lambda)^{3}$. This is the result announced in the introduction.

One might be concerned that higher order contributions to the potential will become important if the first two terms are comparable to one another. A simple analysis shows that the coefficients of the next order terms are generically of size $c_{3} \sim a^{3} \Lambda^{7}$. Compared to the first two terms, these are suppressed by $a \Lambda$, and can be ignored, except for the possibility of a small region of width $\Delta m_{0} \sim a^{4}$ in which all three terms could cancel. Similarly, an $a^{2} \Lambda^{2}$ contribution to $c_{1}$ will shift $m^{\prime}$ by $O\left(a^{2}\right)$ but will not otherwise change the analysis.

To determine the condensate $\Sigma_{0}$ we must minimize the potential energy (4.6). Writing $\Sigma=A+i \mathbf{B} \cdot \boldsymbol{\sigma}$ with $A^{2}+\mathbf{B}^{2}=1$, the potential becomes

$$
\mathcal{V}_{\chi}=-c_{1} A+c_{2} A^{2}
$$

where the parameter $A$ is constrained to lie between -1 and +1 inclusive. Note that the $S U(2)_{V}$ group action, in which $L=R$ in (4.4), leaves $A$ invariant and rotates $\mathbf{B}$ by an orthogonal transformation. Hence, when the vacuum state $\Sigma_{0}=A_{0}+i \mathbf{B}_{\mathbf{0}} \cdot \boldsymbol{\sigma}$ develops a non-zero $\mathbf{B}_{0}$, the flavor symmetry breaks spontaneously to a $U(1)$ subgroup defined by $\exp \left\{i \theta \hat{\mathbf{B}}_{0} \cdot \boldsymbol{\sigma}\right\}$, with $\hat{\mathbf{B}}_{0}$ being the unit vector in the direction of $\mathbf{B}_{0}$. A non-zero value of $\mathbf{B}_{0}$ can occur only when $\left|A_{0}\right|$ is strictly less than one. 
Based on the discussion above, we treat $c_{2}$ as a constant of $O\left(a^{2}\right)$, while $c_{1}$ varies linearly with the bare quark-mass. We do not know the sign of $c_{2}$. It turns out that if $c_{2}$ is positive, we reproduce the properties of the Aoki phase, and so we discuss this case first. The potential is then a parabola with its minimum at $A_{m}=\epsilon$, where we have defined the parameter $\epsilon=c_{1} / 2 c_{2} \sim m^{\prime} /\left(a^{2} \Lambda^{3}\right)$. If this minimum lies outside the range -1 to 1 , then $A_{0}$ is forced to one of the boundary points $\left|A_{0}\right|=1$. This corresponds to $\Sigma_{0}= \pm 1$, and hence the vector symmetry $S U(2)_{V}$ is not spontaneously broken. This situation is illustrated in Fig. 凤. If instead the minimum satisfies $\left|A_{m}\right|<1$, then the vacuum is determined by $A_{0}=A_{m}$ and $\mathbf{B}_{0} \neq 0$, as illustrated in Fig. 3. Since $\mathbf{B}_{0} \neq 0$, flavor symmetry is spontaneously broken to $U(1)$, and the region $-1<\epsilon<1$ thus has the properties of the Aoki phase.

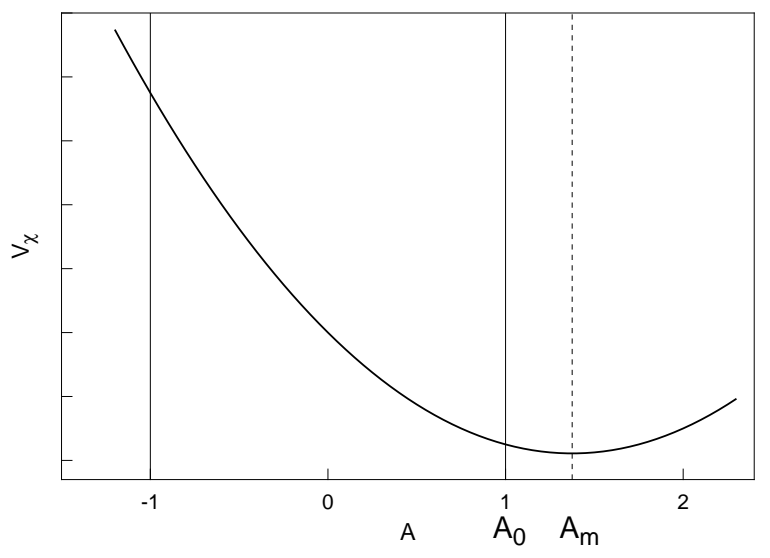

FIG. 2. The potential $\mathcal{V}_{\chi}$ vs. $A$ for $c_{2}>0$ and $\left|A_{m}\right|>1$. The vacuum is at $A_{0}=1$.

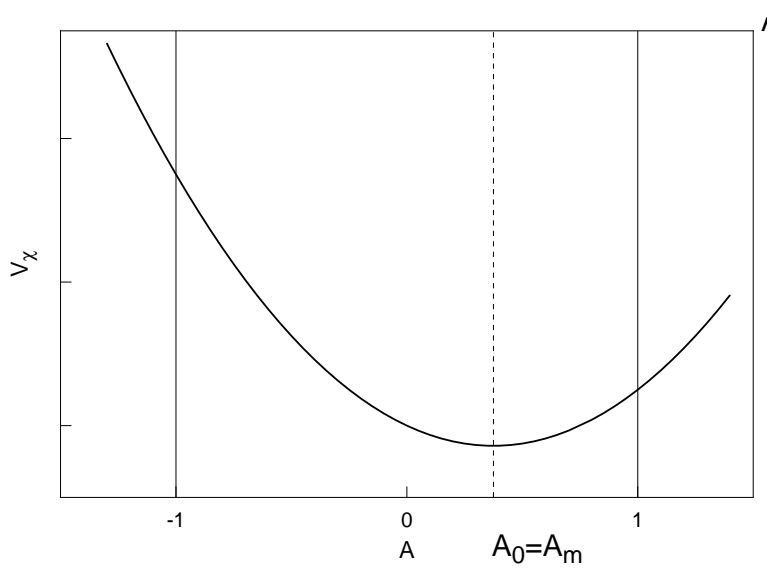

FIG. 3. The potential $\mathcal{V}_{\chi}$ vs. $A$ for $c_{2}>0$ and $\left|A_{m}\right|<1$. The vacuum is at $A_{0}=A_{m}$.

Let us examine the spontaneous flavor breaking in more detail. In the Aoki phase, the direction of $\mathbf{B}_{0}$ is determined by the source term. To make contact with the numerical simulations, we assume a source that aligns $\mathbf{B}_{0}$ in the 3 -direction, and adopt the new parameterization $\Sigma_{0}=\cos \theta_{0}+i \sin \theta_{0} \sigma_{3}$. The angle $\theta_{0}$ is determined by $\epsilon$, as explained above, 


$$
\cos \theta_{0}=\left\{\begin{array}{cr}
-1 & \epsilon \leq-1 \\
\epsilon & -1 \leq \epsilon \leq 1 \\
+1 & 1 \leq \epsilon
\end{array}\right.
$$

The vacuum smoothly interpolates between the two flavor-symmetric values. To determine the pion masses, we expand $\Sigma$ about the condensate (4.5), finding

$$
A=\cos \theta_{0}-\frac{\sin \theta_{0}}{f_{\pi}} \pi_{3}-\frac{\cos \theta_{0}}{2 f_{\pi}^{2}} \sum_{a=1}^{3} \pi_{a}^{2}+\mathcal{O}\left(\pi_{a}^{3}\right) .
$$

The potential becomes

$$
\mathcal{V}_{\chi}=\left\{\begin{array}{cc}
\frac{c_{2}}{f_{\pi}^{2}}\left(1-\epsilon^{2}\right) \pi_{3}^{2}-c_{2} \epsilon^{2}+\mathcal{O}\left(\pi_{a}^{3}\right) & |\epsilon|<1 \\
\frac{c_{2}}{f_{\pi}^{2}}(|\epsilon|-1) \sum_{a} \pi_{a}^{2} & 1 \leq|\epsilon|
\end{array},\right.
$$

and hence the pion masses are

$$
\begin{aligned}
m_{1}^{2}=m_{2}^{2}=0, \quad \frac{m_{3}^{2} f_{\pi}^{2}}{2 c_{2}}=1-\epsilon^{2} \quad \text { for } \quad|\epsilon| \leq 1 \\
\frac{m_{a}^{2} f_{\pi}^{2}}{2 c_{2}}=|\epsilon|-1 \quad \text { for } \quad|\epsilon| \geq 1 .
\end{aligned}
$$

The results are shown in Fig. 4 . We see that the pions $\pi_{1,2}$ are the Goldstone bosons of the broken flavor symmetry within the Aoki phase, and that all three pions are massless on the phase boundaries.

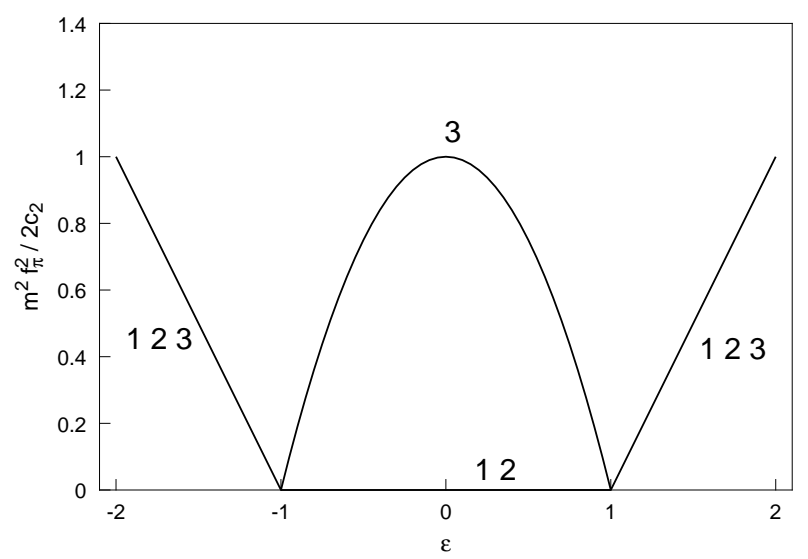

FIG. 4. Pion masses as a function of $\epsilon$ for $c_{2}>0$. The curves are labeled by the flavor of the corresponding pion.

In summary, we have reproduced the phenomenology of the Aoki phases using the chiral Lagrangian. In addition, our calculation makes three predictions:

1. As already mentioned above, for small enough $a$, the width of the Aoki phase should scale as $\Delta m_{0} \sim a^{3}$. This should hold up to logarithmic corrections, which we have ignored throughout. 
2. From (4.14), the mass of the $\pi_{3}$ meson within the Aoki phase is predicted in terms of the form outside the phase. For example, the slope of $m_{3}^{2}$ versus the dimensionless bare mass $m_{0}$ should be a factor of two larger as the phase boundary is approached from within compared to approaching it from without. In both cases, $m_{3} \sim a$ when $\epsilon \sim \mathcal{O}(1)$.

3. The form of the spectral density of the overlap Hamiltonian at zero eigenvalue can be determined. To do this we note that

$$
\left\langle\bar{\psi}_{a} i \gamma_{5} \psi_{b}\right\rangle \propto i\left[\Sigma_{0}-\Sigma_{0}^{\dagger}\right]_{a b} .
$$

On the lattice, the 1.h.s. is proportional to $\rho\left(0 ; m_{0}\right)$, while we have found that the r.h.s. is proportional to $\sin \theta_{0}$, and therefore

$$
\rho\left(0 ; m_{0}\right) \propto \sqrt{1-\epsilon^{2}} \propto m_{3} .
$$

It should be possible to test some of these predictions.

We now return to the possibility that the coefficient $c_{2}$ is negative. The potential is then an inverted parabola, and the extremum $A_{m}$ is an absolute maximum. This means that the vacuum state is always at the edge of the allowed range of $A$, with $\Sigma_{0}=+1$ for $c_{1}>0$ and $\Sigma_{0}=-1$ for $c_{1}<0$. The potential can be written

$$
\mathcal{V}_{\chi}=\frac{\left|c_{2}\right|}{f_{\pi}^{2}}(1+|\epsilon|) \sum_{a=1}^{3} \pi_{a}^{2}+c_{2}-\left|c_{1}\right|+\mathcal{O}\left(\pi_{a}^{3}\right)
$$

Thus the flavor symmetry is not spontaneously broken for any value of $\epsilon$, and all three pions have the same non-zero mass,

$$
\frac{m_{a}^{2} f_{\pi}^{2}}{2\left|c_{2}\right|}=1+|\epsilon|
$$

This situation is illustrated in Fig. 5. Note that $m_{a} \sim a$ for $\epsilon \sim \mathcal{O}(1)$.

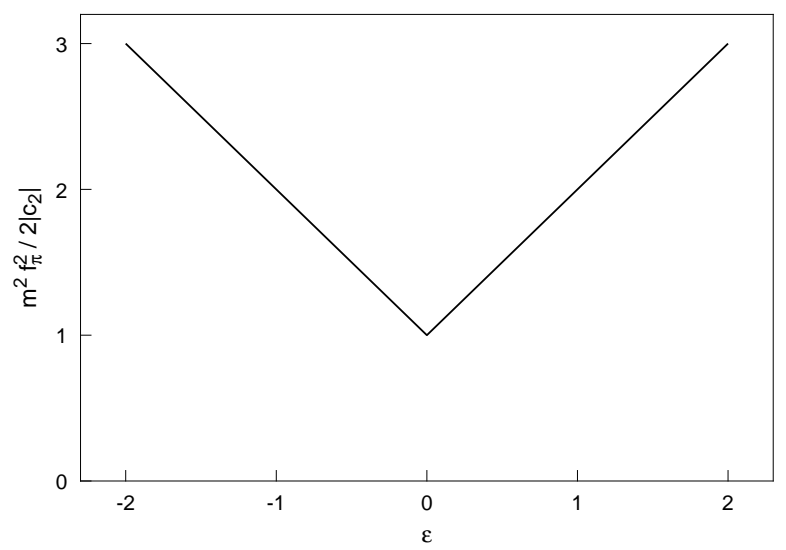

FIG. 5. Pion masses as a function of $\epsilon$ for $c_{2}<0$. 
The previous analysis cannot choose between the $c_{2}>0$ and $c_{2}<0$ cases - for this we must rely on simulations. As noted in Sec. III, all numerical observations to date are consistent with there being spontaneous flavor and parity breaking at non-zero lattice spacing, and hence with $c_{2}$ being positive. Thus it appears that an Aoki phase with a width of order $a^{3}$ will persist until the continuum is reached. There are, however, two caveats that we should mention. First, removing the quenched (or partially quenched) approximation could, in principle, lead to a change in the sign of $c_{2}$. Second, it is logically possible that, for the lattice spacings studied to date, terms of higher order in $a$ are important and conspire with the terms we considered above in such a way that the effective value of $c_{2}$ changes sign as the continuum limit is approached. In this case the Aoki phase would disappear for small enough lattice spacing.

It is interesting to consider in what way the previous analysis changes when one uses improved Wilson fermions. The surprising answer is that the analysis is essentially unaltered, even if one removes all errors proportional to $a$ using non-perturbative improvement conditions. The reason is that the key discretization error is the $O\left(a^{2}\right)$ contribution to $c_{2}$ [see (4.7)], and this remains after improvement. Of course, the sign of the coefficient $c_{2}$ can change, so we cannot predict whether there will be an Aoki phase. What we can say is that

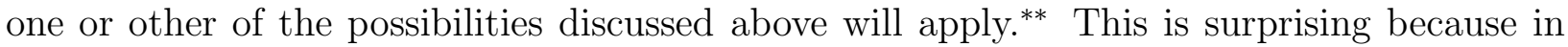
both scenarios the non-vanishing pion masses are proportional to $a$ for $\epsilon \sim O(1)$, while one would have naively expected the masses to be proportional to $a^{2}$. We see nothing wrong with this result, however. In particular, for any fixed physical quark-mass, the discretization errors are of $O\left(a^{2}\right)$ for small enough lattice spacing.

\section{CONCLUSIONS}

We have considered the issue of spontaneous flavor and parity symmetry breaking in lattice QCD, and its relation to chiral symmetry breaking in the continuum. Contrary to the claims of Bitar et al. [6], we have shown that flavor-parity breaking can occur at any non-zero lattice spacing. Indeed, our chiral Lagrangian analysis shows that such symmetry breaking is one of only two options available to the theory as the continuum limit is approached. If this option is chosen, as is indicated by present numerical evidence, then our calculation shows how the breaking of flavor and parity at non-zero lattice spacing goes over smoothly into chiral symmetry breaking in the continuum limit. This is the sense in which we agree with the proposal of Bitar et al. that one can think of flavor-parity breaking on the lattice as corresponding to chiral symmetry breaking in the continuum.

Based on this proposal, Bitar et al. suggest obtaining the continuum chiral condensate, $C_{0}=\langle\bar{u} u\rangle+\langle\bar{d} d\rangle$, in the following way. Calculate the condensate $\left\langle\bar{\psi} i \gamma_{5} \sigma_{3} \psi\right\rangle$ in the infinite volume limit at non-zero lattice spacing; average the result over the Aoki-phase; and then take the continuum limit. Their major point, with which we agree, is that the lattice condensate is a good order parameter, and in particular is free from additive renormalization. It follows

\footnotetext{
**In fact the numerical evidence of Ref. [8] suggests an Aoki phase also for non-perturbatively improved fermions.
} 
from our analysis, however, that one should not average over the Aoki phase, but rather take the maximum value of the lattice condensate within the Aoki phase, and extrapolate this to the continuum limit. If one were to average over the Aoki phase then one would find a value for the continuum condensate too small by a factor of $\pi / 4$.

To understand this, recall that the condensate takes the form $\Sigma_{0}=\cos \theta_{0}+i \sin \theta_{0} \sigma_{3}$ in the Aoki phase, and so

$$
\begin{gathered}
\langle\bar{\psi} \psi\rangle=C_{0} \operatorname{Tr}\left(\frac{\Sigma_{0}+\Sigma_{0}^{\dagger}}{4}\right)=C_{0} \cos \theta_{0}, \\
\left\langle\bar{\psi} i \gamma_{5} \sigma_{3} \psi\right\rangle=C_{0} \operatorname{Tr}\left(\frac{\Sigma_{0}-\Sigma_{0}^{\dagger}}{4 i}\right) \sigma_{3}=C_{0} \sin \theta_{0}
\end{gathered}
$$

Thus at a general position in the Aoki phase, one can obtain continuum condensate from

$$
C_{0}^{2}=\langle\bar{\psi} \psi\rangle^{2}+\left\langle\bar{\psi} i \gamma_{5} \sigma_{3} \psi\right\rangle^{2}
$$

On the lattice, however, one can only measure the second of these condensates, and this equals $C_{0}$ only when $\epsilon=\cos \theta_{0}=0$, i.e. when the lattice condensate takes its maximum value. In other words, the lattice condensate picks out a particular component of the chiral condensate, and only when the former is maximal are the two condensates aligned.

One of the predictions of our analysis is that the width of the symmetry-broken phase grows rapidly as the lattice spacing is increased, $\Delta m_{0} \sim a^{3}$. According to Aoki's picture, there is a critical lattice spacing at which the five "fingers" of the Aoki phase merge into a single phase of width $\Delta m_{0} \sim 6$ (see Fig. 1). It is not clear from numerical evidence at what coupling this occurs. At $g^{2}=1$, in the quenched approximation, Ref. [4 finds evidence for all five fingers, while Ref. [8] finds only a single phase down to $m_{0}=-2$. We stress, however, that either possibility is consistent with our results. Our prediction is that there is some critical coupling below which the Aoki phase $B$ splits into finger-like regions of decreasing width, surrounded by islands of unbroken phase $A$, as in Fig. 1. Once one has entered this region then our other predictions, for the pion masses and the condensate, apply as well.

As explained in Ref. [8], practical applications of domain-wall fermions probably require that the Aoki phase has a small width. Our results strongly suggest that this will be the case for small enough lattice spacing.

\section{ACKNOWLEDGMENTS}

We thank Khalil Bhitar, Rajamani Narayanan, Urs Heller and Larry Yaffe for helpful comments and discussions. This research was supported in part by U.S. Department of Energy grant DE-FG03-96ER40956. 


\section{APPENDIX A: THE INAPPLICABILITY OF THE VAFA-WITTEN THEOREMS}

The condensate (2.3) breaks both flavor and parity, in apparent contradiction with Refs. [11 and 12], respectively. In this appendix we give a brief explanation of why these references do not, in fact, rule out such a condensate.

The argument of Ref. [11] against flavor-breaking in the continuum does not apply for massless fermions because the spectrum of the Dirac operator has a non-vanishing density of zero eigenvalues. As has been discussed in Ref. [6], the relevant operator on the lattice is the overlap Hamiltonian, $\gamma_{5} W\left(-m_{0}\right)$, and the Vafa-Witten argument also does not apply if this has a non-vanishing density of zero eigenvalues, which it does in the Aoki phase. These failures are demonstrated by the explicit calculations of Appendix B.

The loophole in the argument against spontaneous parity-breaking is simply that the analysis says nothing about fermionic bilinears. The bulk of Ref. [12 is devoted to proving that parity-odd bosonic operators do not receive vacuum expectation values. In Footnote 7 , however, Vafa and Witten claim that the theorem can be extended to include fermionic bilinears, since, upon integrating out the fermions, the fermionic operators can be replaced by bosonic operators, and for these their original argumentation applies. The example they consider is the flavor-singlet operator $\bar{q} i \gamma_{5} q$, which is replaced by $X=\operatorname{Tr} i \gamma_{5} S_{A}(x ; x)$, with $S_{A}(x ; y)$ being the fermionic propagator in the background $A$. This extension of their argument is not valid, however. The operator $X$ is either ill-defined if there are fermionic zero modes, or it simply vanishes because the eigenvalues of $\gamma_{5} S_{A}(x ; y)$ come in opposite-sign pairs.

Properly speaking, one must calculate the vacuum expectation value by adding a source, and only take the source strength to zero at the end. The presence of the source regulates the corresponding effective bosonic operator $X$. However, for two flavors of quarks, it also invalidates an essential assumption of Ref. [12], namely that $X$ be imaginary in Euclidean space. Indeed, one can explicitly calculate the form of $X$ using a spectral decomposition of the corresponding fermion operator. For the condensate of interest, (2.3), for which one adds the source (2.2), this calculation is done in Appendix B. We find that the corresponding gluonic operator $X$ is purely real, and so the Vafa-Witten argument does not apply. This point has also been noted in Ref. [2].

It should be noted, however, that Vafa and Witten's argument does still imply that all parity-odd operators composed only of gluon fields do not have vacuum expectation values. This suggests that there exists a modification of parity which acts in the same way as parity

\footnotetext{
${ }^{\dagger}$ For the flavor-singlet parity-odd operator $\bar{q} i \gamma_{5} q$, on the other hand, the effective bosonic operator $X$ in the presence of the source $\mathcal{L}_{\text {source }}=h \bar{q} \exp \left[i \theta \gamma_{5}\right] q$ is not pure real, and the loophole in applying Ref. [12] is more subtle. Note that the source can be transformed into the mass term $h \bar{q} q$ by an axial $U(1)$ transformation, which, because of the axial anomaly, introduces a $\theta \tilde{F} F$ term. The source therefore does not have the usual effect of picking a particular direction for the condensate, but instead selects a different theory characterized by a parity-violating $\theta$-vacuum. This is true even for infinitesimal source strength, and thus Ref. [12] does not imply that the fermionic parity-breaking condensate $\left\langle\bar{q} i \gamma_{5} q\right\rangle$ vanishes.
} 
on the gluon fields, but differently on fermions, and which is unbroken by the condensate in the Aoki phase. Such a transformation does exist: it is the product of parity with a discrete flavor transformation,

$$
P^{\prime}=P \otimes z, \quad z=i \sigma_{1} \in S U(2)
$$

The flavor transformation has no effect on the gluon fields. Note that $\left(P^{\prime}\right)^{2}=-1$ when acting on fermion fields, so that $P^{\prime}$ generates a $Z_{4}$ group. When acting on the bilinear $\bar{\psi} i \gamma_{5} \sigma_{3} \psi$, however, its square is the identity. Note also that other choices of $z$ are possible, but all are related by transformations in the unbroken $U(1)$ flavor subgroup.

\section{APPENDIX B: CALCULATION OF THE CONDENSATES}

In this appendix we compute the condensates $\left\langle\bar{\psi}_{b} \Gamma \psi_{a}\right\rangle$ in both the continuum and on the infinite-volume lattice with Wilson fermions. We work in two-flavor QCD with a common bare mass, and we denote the quark field with flavor index $a$ by $\psi_{a}$. The quantity $\Gamma$ is an appropriately chosen spinor-matrix, which in the continuum can be either the unit matrix $\mathbf{1}$ or $i \gamma_{5}$, while on the lattice we consider only $\Gamma=i \gamma_{5}$. As discussed in Sec. III, the condensates are defined by adding to the Lagrangian the source term

$$
\mathcal{L}_{\text {source }}(\boldsymbol{\theta}, h)=h \bar{\psi} \exp \left[i \boldsymbol{\theta} \cdot \boldsymbol{\sigma} \gamma_{5}\right] \psi,
$$

computing $\left\langle\bar{\psi}_{b} \Gamma \psi_{a}\right\rangle_{h}$ for non-zero $h$, and then taking the $h \rightarrow 0^{+}$limit. We will let $\theta$ denote the modulus of $\boldsymbol{\theta}$, while $\hat{\boldsymbol{\theta}}$ will represent a unit vector in the direction of $\boldsymbol{\theta}$. Without loss of generality we can take $h>0$ and $0 \leq \theta<\pi$ (so that $\sin \theta \geq 0)$.

\section{The continuum theory}

We first consider the massless continuum theory with the fermionic Lagrangian $\mathcal{L}_{f}=$ $\bar{\psi}_{a} \not D \psi_{a}$. It will be convenient to place the system in a finite box of four-volume $V_{4}$, so that the Euclidean (anti-Hermitian) Dirac-operator $\not D$ has discrete eigenvalues $\pm i \lambda_{n}$ with $\lambda_{n} \geq 0$, and at the end of the calculation the infinite-volume limit followed by the $h \rightarrow 0^{+}$limit will be taken. For simplicity, let us first work out the flavor diagonal case $\hat{\boldsymbol{\theta}}=\hat{\boldsymbol{z}}$ with arbitrary $\theta$. After performing the fermionic path integrals, the condensates in the presence of the source can be written

$$
\left\langle\bar{\psi}_{b} \Gamma \psi_{a}\right\rangle_{h}=\int \mathcal{D} A \exp \left\{-S_{g}[A]\right\}[\operatorname{det}(\not D+h)]^{2}\left\langle\bar{\psi}_{b} \Gamma \psi_{a}\right\rangle_{A, h}
$$

with $S_{g}[A]$ being the pure gluonic Euclidean action, and where the fixed-background condensates take the form

$$
\left\langle\bar{\psi}_{b} \Gamma \psi_{a}\right\rangle_{A, h}=\frac{1}{V_{4}} \int d^{4} x\left\langle\bar{\psi}_{b} \Gamma \psi_{a}(x)\right\rangle_{A, h}=-\frac{1}{V_{4}} \operatorname{Tr} \frac{\Gamma \delta_{a b}}{\not D+h \exp \left\{i \theta \gamma_{5} \sigma_{3 a b}\right\}} .
$$

The trace includes a sum over color, spin, and space-time, and it can be written 


$$
\operatorname{Tr} \frac{\Gamma}{\not D+h \exp \left\{ \pm i \theta \gamma_{5}\right\}}=\sum_{\lambda_{n} \geq 0} \frac{2 h}{\lambda_{n}^{2}+h^{2}} \times\left\{\begin{aligned}
\cos \theta & \text { for } \Gamma=1 \\
\pm \sin \theta & \text { for } \Gamma=i \gamma_{5}
\end{aligned}\right.
$$

where, in computing the fermionic determinant in (B2) and the trace $(\mathbb{B} 4)$, we have used the fact that

$$
\begin{array}{rll}
\not D+m_{1}+i \mu_{1} \gamma_{5} & \text { has eigenvalues } & \Lambda_{n}^{ \pm}=m_{1} \pm i \sqrt{\lambda_{n}^{2}+\mu_{1}^{2}} \\
\gamma_{5}\left(\not D+m_{1}+i \mu_{1} \gamma_{5}\right) & \text { has eigenvalues } & \Lambda_{n}^{ \pm}=i \mu_{1} \pm \sqrt{\lambda_{n}^{2}+m_{1}^{2}}
\end{array}
$$

Since we are working with two-flavor QCD, the fermionic determinant is real, positive, and $\theta$-independent.

In infinite volume, the eigenvalues of the Hermitian operator $-i \not D$ become continuous and are described by a spectral density per unit four-volume $\tilde{\rho}_{A}(\lambda)$. Since $-i \not D$ and $\gamma_{5}$ anticommute, the eigenstates of $-i \not D$ come in pairs $\phi_{\lambda}(x)$ and $\gamma_{5} \phi_{\lambda}(x)$ with eigenvalues $\lambda$ and $-\lambda$ respectively. Therefore, $\tilde{\rho}_{A}(\lambda)$ is symmetric in $\lambda$ and we can write

$$
\lim _{h \rightarrow 0^{+}} \lim _{V_{4} \rightarrow \infty} \frac{1}{V_{4}} \sum_{n} \frac{2 h}{\lambda_{n}^{2}+h^{2}}=\lim _{h \rightarrow 0^{+}} \int_{-\infty}^{\infty} d \lambda \tilde{\rho}_{A}(\lambda) \frac{h}{\lambda^{2}+h^{2}}=\pi \tilde{\rho}_{A}(0),
$$

where we have used the relation

$$
\lim _{h \rightarrow 0^{+}} \frac{1}{\lambda \pm i h}=\mp i \delta(\lambda)+P \frac{1}{\lambda},
$$

with $P \frac{1}{\lambda}$ denoting the principal part under the $\lambda$ integral. The principal-part contribution vanishes, however, because of the symmetry in $\lambda$. In the flavor diagonal case we thus find

$$
\lim _{h \rightarrow 0^{+}}\left\langle\bar{\psi}_{b} \Gamma \psi_{a}\right\rangle_{A, h}=-\pi \tilde{\rho}_{A}(0) \times\left\{\begin{array}{ll}
\cos \theta \delta_{a b} & \text { for } \Gamma=\mathbf{1} \\
\sin \theta \sigma_{3 a b} & \text { for } \Gamma=i \gamma_{5}
\end{array} .\right.
$$

The condensate in the presence of the source (B1) with an arbitrary flavor orientation $\hat{\boldsymbol{\theta}}$ can be obtained from (B8) by an appropriation spinor-rotation. If we write $\hat{\boldsymbol{\theta}}=(\sin \alpha \cos \beta, \sin \alpha \sin \beta, \cos \alpha)$ with $\hat{\boldsymbol{\Theta}}=(\sin (\alpha / 2) \cos \beta, \sin (\alpha / 2) \sin \beta, \cos (\alpha / 2))$, then $R \equiv i \hat{\boldsymbol{\Theta}} \cdot \boldsymbol{\sigma}$ satisfies

$$
R \sigma_{3} R^{\dagger}=\hat{\boldsymbol{\theta}} \cdot \boldsymbol{\sigma}
$$

and we see that $R$ is a spin-1/2 representation of the 3 -dimensional rotation from $\hat{\boldsymbol{z}}$ to $\hat{\boldsymbol{\theta}}$. As $\gamma_{5}$ does not act in the flavor space and since its square is unity, the matrix $R$ also transforms the diagonal source with $\hat{\boldsymbol{\theta}}=\hat{\boldsymbol{z}}$ to the general source (B1) using the same transformation as (B9), i.e.

$$
R \exp \left[i \theta \sigma_{3} \gamma_{5}\right] R^{\dagger}=\exp \left[i \boldsymbol{\theta} \cdot \boldsymbol{\sigma} \gamma_{5}\right] .
$$

Using $(\overline{\mathrm{B} 10})$, the diagonal condensate $(\overline{\mathrm{B} 8})$ can be rotated to a general orientation $\hat{\boldsymbol{\theta}}$ to produce

$$
\begin{aligned}
\left\langle\bar{\psi}_{b} \psi_{a}\right\rangle_{A} & =-\pi \tilde{\rho}_{A}(0) \cos \theta \delta_{a b} \\
\left\langle\bar{\psi}_{b} i \gamma_{5} \psi_{a}\right\rangle_{A} & =-\pi \tilde{\rho}_{A}(0) \sin \theta[\hat{\boldsymbol{\theta}} \cdot \boldsymbol{\sigma}]_{a b} .
\end{aligned}
$$


The condensates $\left\langle\bar{\psi}_{b} \Gamma \psi_{a}\right\rangle$ can now be obtained with the help of $(\mathbb{B} 2)$, and they take the same form as (B11), except that one uses the gauge averaged spectral density

$$
\tilde{\rho}(\lambda)=\int \mathcal{D} A \exp \left\{-S_{g}[A]\right\}[\operatorname{det} \not D]^{2} \tilde{\rho}_{A}(\lambda) .
$$

Equation (3.2) now follows.

It is also interesting to consider the condensates for a non-zero common mass $m$ with fermionic Lagrangian $\mathcal{L}_{f}=\bar{\psi}_{a}(\not D+m) \psi_{a}$. As with the massless case in (B6 $)$, a condensate before the $h \rightarrow 0^{+}$limit has been taken can be expressed as a spectral integral of some rational function. The numerators of the scalar and pseudo-scalar condensates are proportional to $m+h \cos \theta$ and $h \sin \theta$ respectively, while the denominators of both condensates are $\lambda^{2}+h^{2}+m^{2}+2 m h \cos \theta$. The non-zero mass $m$ renders the $h \rightarrow 0^{+}$limit safe for all eigenvalues $\lambda$, thereby producing the $\theta$-independent expressions

$$
\begin{aligned}
\left\langle\bar{\psi}_{b} \psi_{a}\right\rangle_{A} & =-\int_{-\infty}^{\infty} d \lambda \tilde{\rho}_{A}(\lambda) \frac{m}{\lambda^{2}+m^{2}} \delta_{a b} \\
\left\langle\bar{\psi}_{b} i \gamma_{5} \psi_{a}\right\rangle_{A} & =0 .
\end{aligned}
$$

Of course, taking the $m \rightarrow 0$ limit first would give (B11), and we see that the $m \rightarrow 0$ and $h \rightarrow 0^{+}$limits do not commute. Note, however, that (B13a) is consistent with the fact that flavor is not spontaneously broken for massive quarks [11], since it implies that $\left\langle\bar{\psi} \sigma_{3} \psi\right\rangle=0$, while $(\mathrm{B} 13 \mathrm{~b})$ is consistent with there being no spontaneous flavor or parity breaking.

\section{The infinite-volume lattice theory}

While much of the corresponding calculation on the infinite-volume lattice with Wilson fermions is similar to the continuum case, there are some key distinctions which we outline in this section. For one thing, the Wilson-Dirac operator $W\left(m_{0}\right)$ has complex eigenvalues and therefore does not possess a spectral density. This suggests that one tries to relate the condensates to the overlap Hamiltonian $H\left(-m_{0}\right)=\gamma_{5} W\left(m_{0}\right)$, which is Hermitian and therefore has eigenvalues constrained to lie along the real axis. For a fixed background configuration $U$, we will write the discrete finite-volume eigenvalues of $\gamma_{5} W\left(m_{0}\right)$ as $\lambda_{n}\left(m_{0} ; U\right)$, while in infinite volume the corresponding spectral density will be denoted by $\rho_{U}\left(\lambda ; m_{0}\right)$. Therefore, the fermionic Lagrangian for the quarks $\psi_{a}$ with a common bare mass $m_{0}$ can be written $\mathcal{L}_{f}=\bar{\psi}_{a} W\left(m_{0}\right) \psi_{a}=\psi_{a}^{\prime} H\left(-m_{0}\right) \psi_{a}$, where we now consider $\psi_{a}$ and $\psi_{a}^{\prime}=\bar{\psi}_{a} \gamma_{5}$ as the independent Grassmann variables. While the Wilson term explicitly breaks the chiral symmetry, there is nonetheless an exact $S U(2)$ flavor symmetry. We should point out that, on the lattice, we only calculate $\left\langle\bar{\psi}_{b} \Gamma \psi_{a}\right\rangle$ with $\Gamma=i \gamma_{5}$. Unlike the continuum, the condensate with $\Gamma=1$ is not proportional to the spectral density, since the eigenvectors of $\gamma_{5} W\left(m_{0}\right)$ with opposite-sign eigenvalues are not connected by $\gamma_{5}$.

We first consider the flavor diagonal case $\hat{\boldsymbol{\theta}}=\hat{\boldsymbol{z}}$ with arbitrary $\theta$. As in the continuum, we place the system in a finite box of four-volume $V_{4}$, calculate the condensate with source (B1), and then take the infinite-volume limit followed by the $h \rightarrow 0^{+}$limit. The condensate

$\left\langle\bar{\psi}_{b} i \gamma_{5} \psi_{a}\right\rangle_{h}$ vanishes when $\theta=0$, and we therefore concentrate on $\theta>0$. After performing the fermionic path integrals over $\psi$ and $\psi^{\prime}=\bar{\psi} \gamma_{5}$, we find 


$$
\left\langle\bar{\psi}_{b} i \gamma_{5} \psi_{a}\right\rangle_{h}=\int \mathcal{D} U \exp \left\{-S_{g}[U]\right\}\left|\operatorname{det}\left[\gamma_{5} W\left(m_{0}\right)+h \gamma_{5} \exp \left\{i \theta \gamma_{5}\right\}\right]\right|^{2}\left\langle\bar{\psi}_{b} i \gamma_{5} \psi_{a}\right\rangle_{U, h}
$$

with $S_{g}[U]$ being the Euclidean Wilson action, and where the background condensates take the form

$$
\left\langle\bar{\psi}_{b} i \gamma_{5} \psi_{a}\right\rangle_{U, h}=-\frac{1}{V_{4}} \operatorname{Tr} \frac{i \delta_{a b}}{\gamma_{5} W\left(m_{0}\right)+h \gamma_{5} \exp \left\{i \theta \gamma_{5} \sigma_{3 a b}\right\}},
$$

with the trace including a sum over spin, color, and space-time. Note that in two-flavor QCD, the fermionic determinant factors are real and positive, although unlike in the continuum there is explicit $\theta$-dependence at non-zero $h$. Using the chiral basis for the gamma-matrices, in which $\gamma_{5}=\operatorname{diag}(1,-1)$, the overlap Hamiltonian takes the general form [17]

$$
\mathrm{H}\left(-m_{0}\right)=\gamma_{5} W\left(m_{0}\right)=\left(\begin{array}{cc}
\mathrm{B}+m_{0} & \mathrm{C} \\
\mathrm{C}^{\dagger} & -\mathrm{B}-m_{0}
\end{array}\right),
$$

where the two-component spinor operator $C$ is a discretized chiral Dirac operator, and $B$ arises from the Wilson term. From this expression, the operator appearing in the denominator of (B15) can be written

$$
\begin{aligned}
\gamma_{5} W\left(m_{0}\right)+h \gamma_{5} \exp \left\{i \theta \gamma_{5} \sigma_{3 a b}\right\} & =\gamma_{5} W(M)+i h \sin \theta \sigma_{3 a b} \\
& \text { with } M=m_{0}+h \cos \theta,
\end{aligned}
$$

and it therefore has eigenvalues $\lambda_{n}(M ; U)+i h \sin \theta \sigma_{3 a b}$. The infinite-volume limit of the trace thus becomes

$$
\lim _{V_{4} \rightarrow \infty} \frac{1}{V_{4}} \operatorname{Tr} \frac{i}{\gamma_{5} W\left(m_{0}\right)+h \gamma_{5} \exp \left\{i \theta \gamma_{5} \sigma_{3 a b}\right\}}=\int_{-\infty}^{\infty} d \lambda \rho_{U}(\lambda ; M) \frac{i}{\lambda+i h \sin \theta \sigma_{3 a b}} .
$$

Recall that in the continuum, the eigenstates of $-i \not D$ with opposite-sign eigenvalues were connected by $\gamma_{5}$, and this rendered the spectral density $\tilde{\rho}_{A}(\lambda)$ symmetric. This is not true on the lattice, and the spectral density $\rho_{U}\left(\lambda ; m_{0}\right)$ is not symmetric in $\lambda$. Nonetheless, if $U^{\prime}$ is the parity conjugate of the configuration $U$, then $\rho_{U^{\prime}}\left(\lambda ; m_{0}\right)=\rho_{U}\left(-\lambda ; m_{0}\right)$ [17]. We can thus form a symmetric spectral density by averaging over parity conjugated configurations, which we implicitly assume has been done throughout the following.

All that remains now is to take the $h \rightarrow 0^{+}$limit. At non-zero lattice spacing, the spectral density $\rho_{U}\left(\lambda ; m_{0}+h \cos \theta\right)$ uniformly converges to $\rho_{U}\left(\lambda ; m_{0}\right)$ as $h$ vanishes, and therefore the limit may be brought inside the integral and allowed to act separately upon each term in the integrand. As $\sin \theta$ is strictly positive, it may be absorbed into a redefinition of $h$ in the denominator of (B18), and therefore (B7) gives the $\theta$-independent result $\left\langle\bar{\psi}_{b} i \gamma_{5} \psi_{a}\right\rangle_{U}=-\pi \rho_{U}\left(0 ; m_{0}\right) \sigma_{3 a b}$. Remembering that the condensate vanishes when $\theta=0$, and upon rotating to an arbitrary flavor direction specified by $\hat{\boldsymbol{\theta}}$, we therefore find

\footnotetext{
${ }_{\ddagger}$ In the continuum, there is a problem with uniform convergence for massless quarks, but this will dealt with at the end of this section.
} 


$$
\left\langle\bar{\psi}_{b} i \gamma_{5} \psi_{a}\right\rangle_{U}=\left\{\begin{array}{cc}
-\pi \rho_{U}\left(0 ; m_{0}\right)[\hat{\boldsymbol{\theta}} \cdot \boldsymbol{\sigma}]_{a b} & \theta>0 \\
0 & \theta=0
\end{array},\right.
$$

with the gauge-averaged condensates $\left\langle\bar{\psi}_{b} i \gamma_{5} \psi_{a}\right\rangle$ taking the same form, except that one uses the gauge-averaged spectral density

$$
\rho\left(\lambda ; m_{0}\right)=\int \mathcal{D} U \exp \left\{-S_{g}[U]\right\}\left[\operatorname{det} \gamma_{5} W\left(m_{0}\right)\right]^{2} \rho_{U}\left(\lambda ; m_{0}\right) .
$$

Equation (3.3) immediately follows. It can be shown that the gap closes, thereby rendering $\rho_{U}\left(0 ; m_{0}\right)$ non-zero, only when the dimensionless bare mass $m_{0}$ is negative [17].

While the calculational techniques presented in this section are not the most natural for the continuum, one could nonetheless follow the same formal steps that led up to (B18). If the $h \rightarrow 0^{+}$limit can be taken inside the integral, then for quarks of physical mass $m$ (and for non-zero $\theta$ ), one obtains the $\theta$-independent continuum result $\left\langle\bar{\psi}_{b} i \gamma_{5} \psi_{a}\right\rangle_{A}=-\pi \rho_{A}(0 ; m)[\hat{\boldsymbol{\theta}} \cdot \boldsymbol{\sigma}]_{a b}$, with $\rho_{A}(\lambda ; m)$ being the spectral density for the Hermitian operator $\gamma_{5}(\not D+m)$. When $m$ is non-zero, we see from $(2.4)$ that $\rho_{A}(0 ; m)=0$, and the condensate therefore vanishes. This agrees with with $(\mathrm{B} 13 \mathrm{~b})$, which was obtained using the methods of the previous section. However, if $m$ vanishes from the start, from (2.4) it is apparent that even though $\rho_{A}(\lambda ; h)$ converges to $\rho_{A}(\lambda ; 0)$ as $h \rightarrow 0$, it does not do so uniformly. Thus, we may not interchange the spectral integral and the $h \rightarrow 0^{+}$limit in (B18), and the calculational techniques of this section break down. In this case, the methods of the previous section must be used and (B11b) is the correct expression for the condensate.

[1] S. Aoki, Phys. Rev. D30 (1984) 2653; Phys. Rev. Lett. 57 (1986) 3136; Prog. Theor. Phys. 122 (1996) 179, hep-lat/9509008.

[2] S. Aoki and A. Gocksch, Phys. Lett. B231 (1989) 449; B243 (1990) 409; Phys. Rev. D45 (1992) 3845.

[3] S. Aoki, A. Ukawa, and T. Umemura, Phys. Rev. Lett. 76 (1996) 873, hep-lat/9508008; Nucl. Phys. B (Proc. Suppl.) 47 (1996) 511; S. Aoki, T. Kaneda, A. Ukawa, and T. Umemura, Nucl. Phys. B (Proc. Suppl.) 53 (1997) 438, hep-lat/9612010; S. Aoki, Nucl. Phys. B (Proc. Suppl.) 60A (1998) 206, hep-lat/9707020.

[4] S. Aoki, T. Kaneda and A. Ukawa, Phys. Rev. D56 (1997) 1808, hep-lat/9612019.

[5] K. Bitar, Nucl. Phys. B (Proc. Suppl.) 63 (1998) 829, hep-lat/9709086.

[6] K. Bitar, U. Heller, and R. Narayanan, Phys. Lett. B418 (1998) 167, hep-th/9710052.

[7] R. Edwards, U. Heller, R. Narayanan, and R. Singleton. Jr., hep-lat/9711029.

[8] R.G. Edwards, U.M. Heller and R. Narayanan, hep-lat/9802016.

[9] M. Creutz, hep-lat/9608024.

[10] T. Banks and A. Zaks, Nucl. Phys. B196 (1982) 189.

[11] C. Vafa and E. Witten, Nucl. Phys. B234 (1984) 173.

[12] C. Vafa and E. Witten, Phys. Rev. Lett. 53 (1984) 535.

[13] T. Banks and A. Casher, Nucl. Phys. B169 (1980) 103.

[14] K. Symanzik, Nucl. Phys. B226 (1983) 187 and 205. 
[15] M. Lüscher, S. Sint, R. Sommer and P. Weisz, Nucl. Phys. B478 (1996) 365, hep-lat/9605038.

[16] C. Dawson et al., Nucl. Phys. B (Proc.Suppl) 63 (1998) 877, hep-lat/9710027.

[17] R. Narayanan and H. Neuberger, Phys. Rev. Lett. 71 (1993) 3251, hep-lat/9308011; Nucl. Phys. B443 (1995) 305, hep-th/9411108. 